\title{
Indikation zur Anwendung von Blutprodukten
}

Einen Überblick über die Indikationen für die verschiedenen Blutprodukte fasst Tab. 35.1 zusammen.

Ein wichtiger Grund dafür, dass die Vollblutspenden heute wenige Stunden nach der eigentlichen Spende aufgetrennt werden, liegt darin, dass die verschiedenen Komponenten unterschiedliche ideale Lagerungsbedingungen haben, und dass sie eine unterschiedliche Haltbarkeit haben. Darüber hinaus soll der Körper des Patienten nicht durch die Transfusion nicht benötigter oder unter Umständen kontraproduktiver Blutbestandteile belastet werden.

Tab. 35.1 Indikationen für die verschiedenen Blutprodukte

\begin{tabular}{l|l}
\hline Blutprodukt & Indikation \\
\hline Erythrozytenkonzentrat & - Mangel an Sauerstoffträgern \\
\hline Bestrahltes & - Mangel an Sauerstoffträgern \\
Erythrozytenkonzentrat & - Immunsuppression \\
\hline Plasma & - Mangel an Gerinnungsfaktoren/Störung \\
& der plasmatischen Gerinnung \\
& - nicht nur einfacher Mangel an Volumen \\
\hline Thrombozytenkonzentrat & - Mangel an Thrombozyten/Störung in der \\
& zellulären Phase der Gerinnung \\
\hline Thrombozytenpool & - Mangel an Thrombozyten/Störung in der \\
& zellulären Phase der Gerinnung \\
\hline Granulozytenkonzentrat & - Störungen der Immunabwehr \\
& - schwere Infekte \\
\hline
\end{tabular}

